\title{
3-Lie Superalgebras Induced by Lie Superalgebras
}

\author{
Viktor Abramov \\ Institute of Mathematics and Statistics, University of Tartu, 50409 Tartu, Estonia; viktor.abramov@ut.ee; \\ Tel.: +372-737-5872
}

Received: 21 November 2018; Accepted: 31 January 2019; Published: 11 February 2019

\begin{abstract}
We show that given a Lie superalgebra and an element of its dual space, one can construct the 3-Lie superalgebra. We apply this approach to Lie superalgebra of $(m, n)$-block matrices taking a supertrace of a matrix as the element of dual space. Then we also apply this approach to commutative superalgebra and the Lie superalgebra of its derivations to construct 3-Lie superalgebra. The graded Lie brackets are constructed by means of a derivation and involution of commutative superalgebra, and we use them to construct 3-Lie superalgebras.
\end{abstract}

Keywords: Lie superalgebra; supertrace; commutative superalgebra; 3-Lie superalgebra

MSC: 17B60; 17B66

\section{Introduction}

A generalization of Hamiltonian mechanics, in which a ternary analog of Poisson bracket appears in a natural way, was proposed by Nambu in [1]. In this generalization of Hamiltonian mechanics, the right-hand side of analog of Hamilton equation is the ternary bracket of functions and two of these three functions play role of Hamiltonians. The ternary bracket at the right-hand side of analog of Hamilton equation is called a Nambu-Poisson bracket. Filippov in [2] proposed a notion of $n$-Lie algebra, which can be considered as an extension of the concept of binary Lie bracket to $n$-ary brackets. The basic component of a notion of $n$-Lie algebra, proposed by Filippov, is the generalization of Jacobi identity, which is now called Filippov-Jacobi or fundamental identity. Later, a study of the algebraic properties of Nambu-Poisson bracket showed that it is skew-symmetric, satisfies the Leibniz rule for a product of two functions and it also satisfies the Filippov-Jacobi (fundamental) identity. Thus, it turned out that from an algebraic point of view, a generalization of Hamiltonian mechanics, proposed by $\mathrm{Nambu}$, as well as the notion of $n$-Lie algebra, proposed by Filippov, can be considered as an extension of the notion of binary Lie bracket to brackets with $n$ arguments, based on generalized Jacobi identity. An excellent survey on $n$-ary algebras and their applications in theoretical physics can be found in [3].

An important aspect of the generalized Hamiltonian mechanics proposed by Nambu is quantization. The problem of quantization of generalized Hamiltonian mechanics was studied in several papers [4-6]. Particularly in the paper [4] the authors proposed the ternary skew-symmetric bracket for $N$ th order matrices, constructed by means of the commutator of two matrices and the trace of a matrix, and proved that this ternary skew-symmetric bracket satisfies the Filippov-Jacobi identity. Hence the Lie algebra of $N$ th order matrices, endowed with the ternary skew-symmetric bracket, proposed in [4], is the matrix 3-Lie algebra. Later this approach was extended to $n$-Lie algebras and it was shown that any $n$-Lie algebra with an analog of a trace induces $(n+1)$-Lie algebra, whose $(n+1)$-ary Lie bracket is constructed by means of a Lie bracket of $n$-Lie algebra and an analog of a trace $[7,8]$.

The concept of $n$-Lie algebra can be extended to Lie superalgebras with due regard to degrees of elements of vector superspace, and this leads to the concept of a $n$-Lie superalgebra. In $[9,10]$ it was shown that the method of $(n+1)$-Lie algebras induced by $n$-Lie algebras can be extended to $n$-Lie 
superalgebras by means of an analog of supertrace. Particularly it was proved that Lie superalgebra $\mathfrak{g l}(m, n)$ of $(m, n)$-block matrices induces the 3-Lie superalgebra if we endow it with a graded ternary bracket, constructed with the help of binary graded commutator of $\mathfrak{g l}(m, n)$ and the supertrace of $(m, n)$-block matrix. In [11] this approach was extended to 3-ary Hom-Lie superalgebras and the authors showed that given a Hom-Lie superalgebra and its representation one can construct the 3-ary Hom-Lie superalgebra, whose graded ternary Lie bracket is constructed by means of binary graded Lie bracket of Hom-Lie superalgebra and the supertrace. The authors of [11] explored the structures of induced 3-ary Hom-Lie superalgebras such that ideals, center, derived series and central extensions.

In Section 2 we prove Theorem 1, which states that given a Lie superalgebra $\mathfrak{g}$ and an element $\phi$ of its dual space, which vanishes on odd subspace of $\mathfrak{g}$ and satisfies

$$
\phi(x) \phi([y, z])+(-1)^{|x|(|y|+|z|)} \phi(y) \phi([z, x])+(-1)^{|z|(|x|+|y|)} \phi(z) \phi([x, y])=0, x, y, z \in \mathfrak{g},
$$

one can construct 3-Lie superalgebra, whose graded ternary Lie bracket is constructed with the help of binary graded Lie bracket of $\mathfrak{g}$ and $\phi$. This theorem can be applied to Lie superalgebra $\mathfrak{g l}(m, n)$ of $(m, n)$-block matrices if $\phi$ is the supertrace of a matrix. In [9] the approach of induced $n$-Lie algebras was applied to the Lie algebra of vector fields on a smooth manifold and the author constructed ternary Lie bracket of vector fields by means of commutator of vector fields and a differential form, which satisfies conditions similar to (1). In Section 3 we generalize this result to a commutative superalgebra and the Lie superalgebra of its derivations. We also extend the constructions and results of [12] to Lie superalgebras and 3-Lie superalgebras. Particularly we construct graded Lie bracket by means of a derivation, involution of commutative superalgebra. Then we use these Lie superalgebras to construct 3-Lie superalgebras in analogy with approach proposed in Theorem 1.

\section{3-Lie Superalgebras Induced by Lie Superalgebras with an Analog of Supertrace}

In this section, we show that given a Lie superalgebra with analog of the supertrace one can construct a 3-Lie superalgebra.

Let $\mathfrak{g}=\mathfrak{g}_{0} \oplus \mathfrak{g}_{1}$ be a vector superspace. In what follows the degree of a homogeneous vector $x$ will be denoted by $|x|$. A vector superspace $\mathfrak{g}$ is said to be a Lie superalgebra if it is endowed with a graded Lie bracket $\left[\mathfrak{g}_{i}, \mathfrak{g}_{j}\right] \subset \mathfrak{g}_{i+j}$, which is graded skew-symmetric $[x, y]=-(-1)^{|x||y|}[y, x](u, v \in \mathfrak{g})$ and satisfies the graded Jacobi identity

$$
[x,[y, z]]=[[x, y], z]]+(-1)^{|x||y|}[y,[x, z]], \quad x, y, z \in \mathfrak{g} .
$$

One can extend the concept of Lie superalgebra to multiplications with many arguments, that is, $n$-ary multiplications, where $n>2$. In this paper, we consider ternary multiplications. Assume $\mathfrak{g}=\mathfrak{g}_{0} \oplus \mathfrak{g}_{1}$ is a vector superspace. A trilinear mapping $(x, y, z) \in \mathfrak{g} \otimes \mathfrak{g} \otimes \mathfrak{g} \mapsto[x, y, z] \in \mathfrak{g}$ is said to be a graded ternary Lie bracket if $|[x, y, z]|=|x|+|y|+|z|$, it is graded skew-symmetric, i.e.,

$$
[x, y, z]=-(-1)^{|x||y|}[y, x, z], \quad[x, y, z]=-(-1)^{|y||z|}[x, z, y],
$$

and satisfies the graded Filippov-Jacobi identity (Fundamental Identity)

$$
\begin{aligned}
{\left.[x, y,[u, v, w]]=[[x, y, u], v, w]+(-1)^{(|x|+|y|)|u|}[u,[x, y, v], w]\right] } \\
+(-1)^{(|x|+|y|)(|u|+|v|)}[u, v,[x, y, w]] .
\end{aligned}
$$

A vector superspace $\mathfrak{g}$, together with a graded ternary Lie bracket defined on it, is called a 3-Lie superalgebra.

Let $\mathfrak{g}=\mathfrak{g}_{0} \oplus \mathfrak{g}_{1}$ be a Lie superalgebra and [, ] be a graded (binary) Lie bracket of this Lie superalgebra. 
Theorem 1. Let $\phi \in \mathfrak{g}^{*}$. Assume that for any homogenous elements $x, y, z \in \mathfrak{g}$ and for any odd degree element $u \in \mathfrak{g}_{1}$ a linear function $\phi$ satisfies the following conditions:

1. $\phi(x) \phi([y, z])+(-1)^{|x|(|y|+|z|)} \phi(y) \phi([z, x])+(-1)^{|z|(|x|+|y|)} \phi(z) \phi([x, y])=0$,

2. $\phi(u)=0$.

Define

$$
[x, y, z]_{\phi}=\phi(x)[y, z]+(-1)^{|x|(|y|+|z|)} \phi(y)[z, x]+(-1)^{|z|(|x|+|y|)} \phi(z)[x, y]
$$

Then (5) is a graded ternary Lie bracket and $\left(\mathfrak{g},\left[,,_{\phi}\right)\right.$ is a 3-Lie superalgebra.

Proof of Theorem 1. A linear function $\phi$ vanishes on elements of odd degree (second condition), and from this it follows that for various combinations of parities of arguments, the ternary bracket (5) takes on the form

$$
[x, y, z]_{\phi}= \begin{cases}\phi(x)[y, z]+\phi(y)[z, x]+\phi(z)[x, y], & x, y, z \in \mathfrak{g}_{0} \\ \phi(y)[z, x]+\phi(z)[x, y], & x \in \mathfrak{g}_{1}, y, z \in \mathfrak{g}_{0} \\ \phi(z)[x, y], & x, y \in \mathfrak{g}_{1}, z \in \mathfrak{g}_{0} \\ 0, & x, y, z \in \mathfrak{g}_{1} .\end{cases}
$$

Hence the ternary bracket (5) satisfies $|[x, y, z]|=|x|+|y|+|z|$. Next we show that (5) is the graded skew-symmetric. Indeed, if we interchange the positions of two first elements in (5) then we obtain

$$
\begin{aligned}
{[y, x, z]_{\phi} } & =\phi(y)[x, z]+(-1)^{|y|(|x|+|z|)} \phi(x)[z, y]+(-1)^{|z|(|x|+|y|)} \phi(z)[y, x] \\
& =-(-1)^{|x||z|} \phi(y)[z, x]-(-1)^{|y|(|x|+|z|)+|y||z|} \phi(x)[y, z]-(-1)^{|z|(|x|+|y|)+|x||y|} \phi(z)[x, y] \\
& =-(-1)^{|x||y|}\left(\phi(x)[y, z]+(-1)^{|x|(|y|+|z|)} \phi(y)[z, x]+(-1)^{|z|(|x|+|y|)} \phi(z)[x, y]\right) \\
& =-(-1)^{|x||y|}[x, y, z]_{\phi} .
\end{aligned}
$$

The graded skew-symmetry of (5) for other permutations of arguments can be proved similarly. It remains to prove the graded Filippov-Jacobi identity (4). If we expand the graded double ternary brackets in the Filippov-Jacobi identity by applying twice the definition of graded ternary bracket (5), then all terms can be divided into three groups of terms. In the first group of terms we collect all those terms that contain graded double (binary) Lie brackets. For instance, if we open the graded double ternary brackets with the help of (5), then the graded double ternary bracket at the left-hand side of the Filippov-Jacoby identity (4) gives the term

$$
[x, y,[u, v, w]]=\phi(x) \phi(u)[y,[v, w]]+\ldots,
$$

the second term at the right-hand side of the Filippov-Jacoby identity gives the term

$$
\left.(-1)^{(|x|+|y|)|u|}[u,[x, y, v], w]\right]=(-1)^{(|x|+|y|)|u|} \phi(x) \phi(u)[[y, v], w]+\ldots,
$$

and the third term at the right-hand side of the Filippov-Jacoby identity gives the term

$$
(-1)^{(|x|+|y|)(|u|+|v|)}[u, v,[x, y, w]]=(-1)^{(|x|+|y|)(|u|+|v|)} \phi(x) \phi(u)[v,[y, w]]+\ldots
$$

By collecting all these terms on the left-hand side of the Filippov-Jacoby identity, we get the expression

$$
\phi(x) \phi(u)\left([y,[v, w]]-(-1)^{|u|(|x|+|y|)}[[y, v], w]-(-1)^{(|x|+|y|)(|v|+|u|)}[v,[y, w]]\right) .
$$


The structure of this expression is such that $x, u$ generate the product $\phi(x) \phi(u)$ and $y, v, w$ generate the sum of graded double (binary) Lie brackets (with the corresponding sign factors). Bearing in mind precisely this structure, we will say that the expression (8) is generated by the combination $((y, v, w),(x, u))$.

Now, if one of elements $x, u$ (or both) is odd degree element, then the expression (8) vanishes due to the condition 2. If both $x, u$ are even degree elements, i.e., $x, u \in \mathfrak{g}_{0}$, then the expression (8) vanishes due to the graded Jacobi identity.

The remaining terms in the first group of terms (containing graded double binary Lie brackets) are generated by the combinations

$$
\begin{aligned}
& ((x, u, v),(y, w)), \quad((y, u, v),(x, w)) \\
& ((x, u, w),(y, v)), \quad((y, u, w),(x, v)) \\
& ((x, v, w),(y, u))
\end{aligned}
$$

and analogously it can be proved that they all vanish because of either graded Jacoby identity or the condition 2.

The second group of terms includes all terms in which one multiplier is of the form $\phi([]$,$) .$ All these terms vanish due to the condition 1. For example, the left-hand side of the Filippov-Jacobi identity contains the terms

$$
\left((-1)^{\alpha} \phi(u) \phi([v, w])+(-1)^{\beta} \phi(v) \phi([w, u])+(-1)^{\gamma} \phi(w) \phi([u, v])\right)[x, y],
$$

where

$$
\begin{aligned}
& \alpha=(|v|+|w|)(|x|+|y|), \beta=(|v|+|w|)|u|+(|w|+|u|)(|x|+|y|) \\
& \gamma=(|v|+|u|)|w|+(|u|+|v|)(|x|+|y|) .
\end{aligned}
$$

The last group contains those terms that are mutually cancelled. Every pair of mutually cancelled terms is determined by one of the following combinations $(x, y, v),(x, y, w),(x, y, u)$. For example, the combination $(x, y, w)$ determines the expression

$$
\left((-1)^{(|u|+|v|+|w|)(|x|+|y|)}[w,[x, y]]+(-1)^{(|u|+|v|)(|x|+|y|)}[[x, y], w]\right) \phi(u) \phi(v) .
$$

It is easy to see that the terms inside the round brackets cancel each other.

We will call $\left(\mathfrak{g},[,]_{\phi}\right)$ the 3-Lie superalgebra induced by a Lie superalgebra $\mathfrak{g}$ with the help of linear function $\phi$. We can apply Theorem 1 to Lie superalgebra $\mathfrak{g l}(m, n)$ of block matrices

$$
X=\left(\begin{array}{ll}
A & B \\
C & D
\end{array}\right),
$$

where $A$ is a square matrix of order $m, D$ is a square matrix of order $n, C$ is a rectangular $n \times m$-matrix and $B$ is a rectangular $m \times n$-matrix. $X$ is a matrix of even degree if $B=0, C=0$, and $X$ is a matrix of odd degree if $A=0, D=0$. Thus, $\mathfrak{g l}(m, n)=\mathfrak{g l}_{0}(m, n) \oplus \mathfrak{g l}_{1}(m, n)$. The degree of a homogeneous matrix is denoted by $|X|$. The graded Lie bracket is the graded commutator of two matrices, i.e.,

$$
[X, Y]=X \cdot Y-(-1)^{|X||Y|} Y \cdot X
$$

where $X \cdot Y$ is the product of two matrices. For a linear function $\phi \in \mathfrak{g l}^{*}(m, n)$, we can take the supertrace of a matrix

$$
\operatorname{Str}(X)=\operatorname{Tr}(A)-\operatorname{Tr}(D)
$$


Then the conditions 1,2 of Theorem 1 are satisfied, because supertrace vanishes on matrices of odd degree and it also vanishes on graded commutators of matrices, i.e., if $X$ is an odd degree matrix, then $\operatorname{Str}(X)=0$, and $\operatorname{Str}([X, Y])=0$. Thus, according to Theorem 1 the graded ternary commutator

$$
[X, Y, Z]_{S}=\operatorname{Str}(X)[Y, Z]+(-1)^{|X|(|Y|+|Z|} \operatorname{Str}(Y)[Z, X]+(-1)^{|Z|(|X|+|Y|} \operatorname{Str}(Z)[X, Y]
$$

where $X, Y, Z \in \mathfrak{g l}(m, n)$, is the graded ternary Lie bracket and $\left(\mathfrak{g l}(m, n),[, \text {, }]_{S}\right)$ is the 3-Lie superalgebra induced by matrix Lie superalgebra $\mathfrak{g l}(m, n)$ with the help of supertrace.

\section{3-Lie Superalgebras Induced by Commutative Superalgebras}

Let $\mathcal{A}=\mathcal{A}_{0} \oplus \mathcal{A}_{1}$ be a superalgebra. A superalgebra $\mathcal{A}$ is said to be commutative superalgebra if for any two homogeneous elements $u, v \in \mathcal{A}$ it holds $u v=(-1)^{|u||v|} v u$. A degree $m$ derivation (left superderivation [13]) of superalgebra $\mathcal{A}$, where $m$ is either 0 (even degree derivation) or 1 (odd degree derivation), is a linear mapping $\delta: \mathcal{A} \rightarrow \mathcal{A}$ such that it satisfies the graded Leibniz rule

$$
\delta(u v)=\delta(u) v+(-1)^{m|u|} u \delta(v) .
$$

The degree of a derivation $\delta$ will be denoted by $|\delta|$. Hence if $\delta$ is an even degree derivation of superalgebra $\mathcal{A}$, then for any two elements $u, v \in \mathcal{A}$ it satisfies the Leibniz rule

$$
\delta(u v)=\delta(u) v+u \delta(v) .
$$

A superinvolution of a superalgebra $\mathcal{A}$ is an even degree antilinear, i.e.,

$$
(\lambda u+v)^{*}=\bar{\lambda} u^{*}+v^{*}, \lambda \in \mathbb{C}, u, v \in \mathcal{A},
$$

mapping $*: u \in \mathcal{A} \mapsto u^{*} \in \mathcal{A}$ such that $\left(u^{*}\right)^{*}=u$ and $(u v)^{*}=(-1)^{|u||v|} v^{*} u^{*}$. In the case of commutative superalgebra with superinvolution $*$ we have $(u v)^{*}=u^{*} v^{*}$.

Let $\operatorname{Der} \mathcal{A}=\operatorname{Der}_{0} \mathcal{A} \oplus \operatorname{Der}_{1} \mathcal{A}$ be the vector superspace of all derivations of $\mathcal{A}$. The graded commutator $\left[\delta_{1}, \delta_{2}\right]=\delta_{1} \circ \delta_{2}-(-1)^{\left|\delta_{1}\right|\left|\delta_{2}\right|} \delta_{2} \delta_{1}$ turns this vector superspace into the Lie superalgebra. If $\mathcal{A}$ is a commutative superalgebra, then $\operatorname{Der} \mathcal{A}$ has the structure of graded left $\mathcal{A}$-module if one defines the left multiplication $(u \delta)(v)=u(\delta(v))$.

Theorem 2. Let $\mathcal{A}$ be a commutative superalgebra and $\omega: \operatorname{Der} \mathcal{A} \rightarrow \mathcal{A}$ be an even degree homomorphism of graded left $\mathcal{A}$-modules. Define ternary bracket

$$
\left[\delta_{1}, \delta_{2}, \delta_{3}\right]_{\omega}=\omega\left(\delta_{1}\right)\left[\delta_{2}, \delta_{3}\right]+(-1)^{\left|\delta_{1}\right|\left(\left|\delta_{2}\right|+\left|\delta_{3}\right|\right)} \omega\left(\delta_{2}\right)\left[\delta_{3}, \delta_{1}\right]+(-1)^{\left|\delta_{3}\right|\left(\left|\delta_{1}\right|+\left|\delta_{2}\right|\right)} \omega\left(\delta_{3}\right)\left[\delta_{1}, \delta_{2}\right] .
$$

If $\omega$ satisfies the conditions

1. $\omega\left(\delta_{1}\right) \delta_{2}\left(\omega\left(\delta_{3}\right)\right)=(-1)^{\left|\delta_{1}\right|\left|\delta_{2}\right|} \omega\left(\delta_{2}\right) \delta_{1}\left(\omega\left(\delta_{3}\right)\right)$,

2. $\omega\left(\delta_{1}\right) \omega\left(\left[\delta_{2}, \delta_{3}\right]\right)+(-1)^{\left|\delta_{1}\right|\left(\left|\delta_{2}\right|+\left|\delta_{3}\right|\right)} \omega\left(\delta_{2}\right) \omega\left(\left[\delta_{3}, \delta_{1}\right]\right)+(-1)^{\left|\delta_{3}\right|\left(\left|\delta_{1}\right|+\left|\delta_{2}\right|\right)} \omega\left(\delta_{3}\right) \omega\left(\left[\delta_{1}, \delta_{2}\right]\right)=0$,

3. $\omega(\delta)=0$ for any $\delta \in \operatorname{Der}_{1} \mathcal{A}$,

then (18) is a graded ternary Lie bracket and $\left(\operatorname{Der} \mathcal{A},[,,]_{\omega}\right)$ is a 3-Lie superalgebra.

A proof of this theorem is similar to the proof of Theorem 1.

Lemma 1. Let $\mathcal{A}$ be a commutative superalgebra with superinvolution $*$. Let $\delta$ be an even degree derivation of A. Define

$$
\begin{aligned}
& {[u, v]_{\delta}=u \delta(v)-(-1)^{|u||v|} v \delta(u)} \\
& {[u, v]_{*}=u^{*} v-(-1)^{|u||v|} v^{*} u}
\end{aligned}
$$


Then $[,]_{\delta},[,]_{*}$ are graded Lie brackets and $\left(\mathcal{A},[,]_{\delta}\right),\left(\mathcal{A},[,]_{*}\right)$ are Lie superalgebras. Define

$$
[u, v]_{*, \delta}=\left(u-u^{*}\right) \delta(v)-(-1)^{|u||v|}\left(v-v^{*}\right) \delta(u) .
$$

If $(\delta(u))^{*}=-\delta\left(u^{*}\right)$ then (21) is a graded Lie bracket and $\left(\mathcal{A},[,]_{*, \delta}\right)$ is a Lie superalgebra.

Proof of Lemma 1. All three brackets $[,]_{\delta,}[,]_{*,}[,]_{*, \delta}$ have the structure of graded commutators, hence we only need to prove that they satisfy the graded Jacobi identity. In the case of graded commutators $[,]_{\delta},[,]_{*}$ this can be done by straightforward computations. We will prove the graded Jacobi identity only for the graded commutator $[,]_{*, \delta}$, because in this case there will be several additional relations. To simplify notations, we will denote $u^{\prime}=u-u^{*}, v^{\prime}=v-v^{*}$. We will also omit the pair $*, \delta$ in the notation of the graded commutator $[,]_{*, \delta}$ and denote it simply by $[$,$] (this$ simplification will be used only until the end of proof). We have

$$
\begin{aligned}
& {[w,[u, v]]=} \underbrace{w^{\prime} \delta\left(u^{\prime}\right) \delta(v)}_{1}+\underbrace{w^{\prime} u^{\prime} \delta^{2}(v)}_{2}-\underbrace{(-1)^{|u||v|} w^{\prime} \delta\left(v^{\prime}\right) \delta(u)}_{3}-\underbrace{(-1)^{|u||v|} w^{\prime} v^{\prime} \delta^{2}(u)}_{3} \\
&-\underbrace{(-1)^{(w, u v)}\left(u^{\prime} \delta(v)\right)^{\prime} \delta(w)}_{4}+\underbrace{(-1)^{(u, v, w)}\left(v^{\prime} \delta(u)\right)^{\prime} \delta(w)}_{5}, \\
& {[[w, u], v]]=} \underbrace{\left(w^{\prime} \delta(u)\right)^{\prime} \delta(v)}_{1}-\underbrace{(-1)^{|u||w|}\left(u^{\prime} \delta(w)\right)^{\prime} \delta(v)}_{4}-\underbrace{(-1)^{(v, u w)}}_{7} \\
& \underbrace{(-1)^{(v, u w)} v^{\prime} w^{\prime} \delta^{2}(u)}_{v^{\prime} \delta\left(w^{\prime}\right) \delta(u)}+\underbrace{(-1)^{(u, v, w)} v^{\prime} \delta\left(u^{\prime}\right) \delta(w)}_{4}+\underbrace{(-1)^{(u, v, w)} v^{\prime} u^{\prime} \delta^{2}(w)}_{6}, \\
& {[u,[w, v]]=} \underbrace{u^{\prime} \delta\left(w^{\prime}\right) \delta(v)}_{7}+\underbrace{u^{\prime} w^{\prime} \delta^{2}(v)}_{2}-\underbrace{(-1)^{|v||w|} u^{\prime} \delta\left(v^{\prime}\right) \delta(w)}_{-}-\underbrace{(-1)^{|v||w|} u^{\prime} v^{\prime} \delta^{2}(w)}_{5} \\
&-\underbrace{(-1)^{(u, v w)}\left(w^{\prime} \delta(v)\right)^{\prime} \delta(u)}_{9}+\underbrace{(-1)^{(u, v, w)}\left(v^{\prime} \delta(w)\right)^{\prime} \delta(u)}_{3},
\end{aligned}
$$

where the relation (22) is the left-hand side of the graded Jacobi identity and the sum of (23) with (24), multiplied by $(-1)^{|u||w|}$, is the right-hand side of graded Jacobi identity. In (22)-(24) we use the following notations

$$
\begin{aligned}
& (w, u v)=|w||| u|+| w|| v|, \quad(v, u w)=| v|| u|+| v|| w \mid \\
& (u, v w)=|u||v|+|u||w|, \quad(u, v, w)=|u||v|+|u||w|+|v||w| .
\end{aligned}
$$

Making use of the definitions of $I, *$ operations and of the condition $(\delta(u))^{*}=-\delta\left(u^{*}\right)$, we obtain

$$
\begin{aligned}
\left(w^{\prime} \delta(u)\right)^{\prime} & =\left(\left(w-w^{*}\right) \delta(u)\right)^{\prime}=\left(w-w^{*}\right) \delta(u)-\left(\left(w-w^{*}\right) \delta(u)\right)^{*} \\
& =\left(w-w^{*}\right) \delta(u)+\left(w-w^{*}\right)(\delta(u))^{*}=\left(w-w^{*}\right)\left(\delta(u)-\delta\left(u^{*}\right)\right. \\
& =w^{\prime} \delta\left(u^{\prime}\right) .
\end{aligned}
$$

Thus, the first term at the right-hand side of (23) can be written

$$
\left(w^{\prime} \delta(u)\right)^{\prime} \delta(v)=w^{*} \delta\left(u^{*}\right) \delta(v),
$$

and it is easy to see that it cancels with the first term at the right-hand side of (22). We can split the terms of graded Jacobi identity into pairs (this is shown in (22)-(24) by means of integers from 1 to 9) such that terms with the same integer label cancel each other. 
Each element $x$ of algebra with superinvolution $\mathcal{A}$ can be written in the form $x=x_{1}+x_{-1}$, where $x_{1}^{*}=x_{1}, x_{-1}^{*}=-x_{-1}$ and

$$
x_{1}=\frac{1}{2}\left(x+x^{*}\right), x_{-1}=\frac{1}{2}\left(x-x^{*}\right) .
$$

It is worth mentioning that the components $x_{1}, x_{-1}$ have the same degree as $x$, i.e., $\left|x_{1}\right|=\left|x_{-1}\right|=|x|$.

Theorem 3. Let $\mathcal{A}=\mathcal{A}_{0} \oplus \mathcal{A}_{1}$ be a commutative superalgebra, $\chi, \phi, \psi \in \mathcal{A}^{*}$ be linear functions on $\mathcal{A}$. Define ternary brackets

$$
\begin{aligned}
{[x, y, z]_{*} } & =\chi(x)[y, z]_{*}+(-1)^{|x|(|y|+|z|)} \chi(y)[z, x]_{*}+(-1)^{|z|(|x|+|y|)} \chi(z)[x, y]_{*} \\
{[x, y, z]_{\delta} } & =\phi(x)[y, z]_{\delta}+(-1)^{|x|(|y|+|z|)} \phi(y)[z, x]_{\delta}+(-1)^{|z|(|x|+|y|)} \phi(z)[x, y]_{\delta} \\
{[x, y, z]_{*, \delta} } & =\psi(x)[y, z]_{*, \delta}+(-1)^{|x|(|y|+|z|)} \psi(y)[z, x]_{*, \delta}+(-1)^{|z|(|x|+|y|)} \psi(z)[x, y]_{*, \delta}
\end{aligned}
$$

If

1. $\chi\left(x_{1} y_{-1}\right)=(-1)^{|x||y|} \chi\left(y_{1} x_{-1}\right), \forall x, y \in \mathcal{A}$ and $\chi(u)=0, \forall u \in \mathcal{A}_{1}$

2. $\phi\left([x, y]_{\delta}\right)=0, \forall x, y \in \mathcal{A}$ and $\phi(u)=0, \forall x \in \mathcal{A}_{1}$,

3. $(\delta(x))^{*}=-\delta\left(u^{*}\right), \psi\left([x, y]_{\delta}\right)=\psi\left(x^{*} \delta(y)-(-1)^{|x||y|} y^{*} \delta(x)\right), \forall x, y \in \mathcal{A}$ and $\psi(u)=0, \forall u \in \mathcal{A}_{1}$,

then (28)-(30) are graded ternary Lie brackets and $\left(\mathcal{A},[,]_{*}\right),\left(\mathcal{A},[,,]_{\delta}\right),\left(\mathcal{A},[,]_{*, \delta}\right)$ are 3-Lie superalgebras.

Proof of Theorem 3. We begin with the ternary bracket (29). It is proved in Lemma 1 that the graded binary bracket $[,]_{\delta}$ gives the Lie superalgebra structure on a commutative superalgebra $\mathcal{A}$. Next we see that the graded ternary bracket (29) has the same structure as the graded ternary bracket (5) and $\phi$ satisfies the conditions 1,2 of Theorem 1 . Hence it follows from Theorem 1 that (29) is the graded ternary Lie bracket and $\left(\mathcal{A},[,]_{\delta}\right)$ is the 3-Lie superalgebra.

The graded binary Lie bracket (20), constructed with the help of superinvolution, can be written in the form

$$
[x, y]_{*}=\left[x_{1}, y_{1}\right]-\left[x_{-1}, y_{-1}\right]+2\left(x_{1} y_{-1}-(-1)^{\left.x y_{1} x_{-1}\right)},\right.
$$

where $[x, y]=x y-(-1)^{|x||y|} y x$ is the graded commutator. The graded commutativity of algebra $\mathcal{A}$ implies $\left[x_{1}, y_{1}\right]=0,\left[x_{-1}, y_{-1}\right]=0$. Thus

$$
\chi\left([x, y]_{*}\right)=2\left(\chi\left(x_{1} y_{-1}-(-1)^{|x||y|} \chi\left(y_{1} x_{-1}\right)\right)=0 .\right.
$$

Taking into account the assumption $\chi(u)=0, u \in \mathcal{A}_{1}$, we see that the conditions 1,2 of Theorem 1 are satisfied, consequently $(28)$ is the graded ternary Lie bracket and $\left(\mathcal{A},[,,]_{*}\right)$ is the 3-Lie superalgebra.

The graded Lie bracket (21) can be put into the form

$$
[x, y]_{*, \delta}=[x, y]_{\delta}-\left(x^{*} \delta(y)-(-1)^{|x||y|} y^{*} \delta(x)\right)
$$

Thus

$$
\chi\left([x, y]_{*, \delta}\right)=\chi\left([x, y]_{\delta}\right)-\chi\left(x^{*} \delta(y)-(-1)^{|x||y|} y^{*} \delta(x)\right)=0 .
$$

Now from Theorem 1 and Lemma 1 it follows that (30) is the graded ternary Lie bracket and $\left(\mathcal{A},[,]_{*, \delta}\right)$ is the 3-Lie superalgebra.

\section{Discussion}

We would like to discuss the possibilities of realizing graded ternary Lie brackets, constructed in Theorem 3, for the case of concrete commutative superalgebras. As it follows from Theorem 3, in order to construct the graded ternary Lie brackets, we need to find linear functions $\chi, \phi, \psi$, which satisfy the conditions of Theorem 3. One of the conditions requires that every function should vanish on 
odd degree elements of superalgebra. For instance, we can consider the commutative superalgebra of functions on a superspace, constructed with the help of Grassmann algebra with superinvolution. Then an even degree vector field on this superspace can be taken as an even degree derivation of superalgebra. To construct linear functionals $\chi, \phi, \psi$ we can use the Berezin integral. Indeed, it follows from the properties of Berezin integral that it vanishes on odd degree functions in the case of even number of generators of Grassmann algebra, but in the case of Grassmann algebra with superinvolution we have even number of generators. Thus, we can construct a linear functional by combining Berezin integral with a linear functional on even subalgebra of superalgebra of functions, which satisfies the conditions of Theorem 3.

Funding: This research was funded by the institutional funding IUT20-57 of the Estonian Ministry of Education and Research.

Acknowledgments: I wish to express my gratitude to Abdenacer Makhlouf for fruitful discussions on Hom-Lie algebras during my visit as visiting professor to the Département de Mathématiques, Université de Haute-Alsace.

Conflicts of Interest: The author declares no conflict of interest.

\section{References}

1. Nambu, Y. Generalized Hamiltonian Mechanics. Phys. Rev. D 1973, 7, 2405-2412. [CrossRef]

2. Filippov, V.T. $n$-Lie algebras. Sib. Math. J. 1985, 26, 879-891. [CrossRef]

3. Azcárraga, J.A.; Izquierdo, J.M. n-Ary Algebras: A Review with Applications. J. Phys. A Math. Theor. 2010, 43, 293001. [CrossRef]

4. Awata, H.; Li, M.; Minic, D.; Yaneya, T. On the quantization of Nambu brackets. J. High Energy Phys. 2001, 2001, 013. [CrossRef]

5. Dito, G.; Flato, M.; Sternheimer, D.; Takhtajan, L. Deformation quantization and Nambu mechanics. Comm. Math. Phys. 1997, 183, 1-22. [CrossRef]

6. Takhtajan, L. On foundation of generalized Nambu mechanics. Commun. Math. Phys. 1994, 160, $295-315$. [CrossRef]

7. Arnlind, J.; Makhlouf, A.; Silvestrov, S. Construction of $n$-Lie algebras and $n$-ary Hom-Nambu-Lie algebras. J. Math. Phys. 2011, 52, 123502. [CrossRef]

8. Arnlind, J.; Kitouni, A.; Makhlouf, A.; Silvestrov, S. Structure and Cohomology of 3-Lie Algebras Induced by Lie Algebras. In Algebra, Geometry and Mathematical Physics; Proceedings in Mathematics \& Statistics; Springer: Berlin/Heidelberg, Germany, 2014.

9. Abramov, V. Super 3-Lie Algebras Induced by Super Lie Algebras. Adv. Appl. Clifford Algebras 2017, 27, 9-16. [CrossRef]

10. Abramov, V. Matrix 3-Lie superalgebras and BRST supersymmetry. Int. J. Geom. Methods Modern Phys. 2017, 14, 1750160. [CrossRef]

11. Guan, B.; Chen, L.; Sun, B. 3-ary Hom-Lie Superalgebras Induced by Hom-Lie Superalgebras. Adv. Appl. Clifford Algebras 2017, 27, 3063-3082. [CrossRef]

12. Bai, R.; Wu, Y. Constructing 3-Lie algebras. Linear Multilinear Algebra 2015, 63, 2171-2186. [CrossRef]

13. Musson, I.M. Lie Superalgebras and Enveloping Algebras. In Graduate Studies in Mathematics; American Mathematical Society: Providence, RI, USA, 2012; Volume 131. 